\title{
MALOCLUSIONES ASOCIADAS A LA CALIDAD DE VIDA EN POBLACIÓN ANDINA
}

\section{MALOCCLUSIONS ASSOCIATED WITH QUALITY OF LIFE IN ANDEAN POPULATION}

\author{
Mercado-Mamani Sively. ${ }^{1 \mathrm{ac}}$ Mercado-Portal Jorge. ${ }^{2 \mathrm{a}}$ Mamani-Cahuata Luz. ${ }^{2 \mathrm{a}}$ Tapia-Condori Rildo. ${ }^{\text {1b }}$ \\ Carreón-Meza Briefhel. ${ }^{3}$ Zapana-Estrada Nohely. ${ }^{3}$ Ledesma-Herrera Kimberly. ${ }^{4}$ \\ ${ }^{1}$ Universidad Andina Néstor Cáceres Velásquez -Juliaca. Perú. \\ ${ }^{2}$ Universidad Nacional del Altiplano-Puno.Perú \\ 3 Semillero de Investigación Facultad de Odontología - UANCV.Perú \\ ${ }^{4}$ Universidad Católica de Cuenca. Ecuador \\ a Doctor en Salud Pública. \\ b Doctor en Estomatología. \\ ${ }^{\mathrm{c}}$ Especialista en Ortodoncia y Ortopedia Maxilar. \\ *goty20@ hotmail.com
}

\begin{abstract}
Resumen
La Organización Mundial de la Salud (OMS) constató que la salud bucal esta íntegramente relacionada con la calidad de vida de los pacientes (CVRSB), está puede estar íntimamente ligada con su bienestar y salud en general; mientras que las maloclusiones son variaciones anatómicas dentofaciales que afectan la salud del aparato estomatognático especialmente en la parte morfológica, funcional y estética, ya que estas varían según los grupos étnicos, zonas geográficas y método de clasificación, afectando así en las acciones cotidianas de los individuos particularmente en su calidad de vida y bienestar en general. La presente revisión de la literatura está dirigida a la población andina, ya que se ha revisado que clínicamente que dicha población ha sido afectada por diversos tipos de anomalías dentomaxilofaciales. Por tal motivo el objetivo de este artículo es realizar una revisión bibliográfica y verificar como puede afectar las maloclusiones con la calidad de vida de los pacientes; con el fin de demostrar la necesidad de implementar en el Ministerio de sector público los tratamientos ortodónticos en adolescentes de la zona andina afectados de manera psicológica y social.
\end{abstract}

Palabras clave: Maloclusiones, Calidad de Vida.

\begin{abstract}
The World Health Organization (WHO) recognizes the quality of life related to oral health (CVRSB) as an integral part of overall health and wellness, while malocclusions are dentofacial morphological variations can affect the health status optimal of the oral cavity in their morphological, functional or aesthetic aspects that vary according to ethnic groups, geographical areas, classification method, thus affecting the daily activities of individuals, in their well-being or quality of life. We consider this revision addressed to the Andean population, clinically seen that are affected by various types of anomalies dentomaxilofaciales. Therefore the aim of this article is to analyze the impact of malocclusions in the quality of life demonstrating the need to implement the policies health sector orthodontic treatment in adolescents in the Andean area affected in the psychosocial aspect that interferes quality of life.
\end{abstract}

Key words: Malocclusións, Quality of Life.

\section{INTRODUCCIÓN}

Las poblaciones andinas actuales no son homogéneas en su origen y apariencia física. El ambiente andino ha influenciado estas poblaciones de manera importante imprimiéndoles algunas características físicas: talla, desarrollo del tórax, forma del tronco. Un estudio antropométrico realizado en población andina, entre las décadas 50 y 60 , descrito por J.A. Vellard describe: "En la isla del sol, por ejemplo, centro antiguo incaico importante, los mesocéfalos no llegan siquiera al $50 \%$ de la población, braquicéfalos 48,0\%, mesocéfalos $42,0 \%$, dolicocéfalos $10 \%$." ${ }^{1}$ Para Tuncay y Bigerstaff a más de haber una aguda percepción de que 
toda deformidad dentaria es el resultado de una compleja interacción genético-ambiental ligada al factor tiempo. ${ }^{2} \mathrm{La}$ alineación y la intercuspidación son características de la oclusión que ayudan en la función masticatoria, la estética facial y la salud del sistema estomatognático. Las maloclusiones fueron descritas por Edward Angle. Estas alteraciones pueden presentarse en cualquiera de los planos vertical, sagital o transversal. ${ }^{3,4}$ La oclusión ideal es un constructo hipotético, que muy raramente se presenta en los pacientes..$^{5}$ Según la información de la Organización Panamericana de la Salud OPS, la prevalencia de esta condición es superior al $80 \%$ de la población por lo que se presenta como una de las causas más frecuentes de consulta en los servicios de salud dental. ${ }^{6-8}$ Estudios mostraron que en Perú existe un alto índice de maloclusión $(80.8 \%$ ) y un índice muy bajo de oclusión normal (19.2\%), en el departamento de Puno, Galvez en el año de 1975 registró un índice de maloclusión del $95.6 \%$ siendo la oclusión normal un $4.4 \% .{ }^{9}$ La calidad de vida se define como la percepción del individuo con su entorno, es decir en su sistema de cultura y valores en los que vive cada día; especialmente la relación que tiene con sus expectativas, objetivos, padrones y preocupaciones de su vida cotidiana. ${ }^{10}$ La OMS refiere que el concepto de salud necesita incluir aspectos psicosociales, uno de ellos es la calidad de vida, y la relación que esta tiene con las relaciones humanas en la sociedad actual. ${ }^{11}$ La calidad de vida relacionada con la salud bucal se puede medir con distintos instrumentos o cuestionarios según la literatura entre estos tenemos el "Early Childhood Oral Health Impact Scale (ECOHIS) y el Child Oral Impacts on Daily Performances Index (Child-OIDP)" que fueron validados en el idioma español. ${ }^{12,13}$ (Ver la Tabla 1.)

\section{ETIOLOGÍA DE LAS MALOCLUSIONES}

Las etiologías de las maloclusiones son multifactoriales por lo que se deberá tener en cuenta las mezclas entre diferentes orígenes étnicos ya que el individuo pudiera heredar dientes grandes con maxilares pequeños dando como resultado apiñamiento dental o al contrario. Existe interacción entre la herencia y el ambiente. ${ }^{14,15}$

\section{3 ÍNDICES EPIDEMIOLÓGICOS DE LAS MALO- CLUSIONES}

Los índices son coeficientes o proporciones que miden la frecuencia de la enfermedad en una comunidad, pueden también ser útiles para determinar la frecuencia de los grados de severidad de la enfermedad en un grupo de personas. ${ }^{16}$ Jhonson, indica que para evaluar el estado de salud bucal de los pacientes con relación a la enfermedad debe cumplir con los siguientes aspectos: ${ }^{17}$ • Pertinencia: La enfermedad y el índice deberán tener relación. - Confianza: Cuando sea sometido al análisis estadístico es preciso que el índice mantenga su validez para poder confiar. Las conclusiones deben ser seguras. $\bullet$ Significado: A todo aquello que pretende medir el índice debe complementar ideas evidentes.

\section{4 ÍNDICE DE NECESIDAD DE TRATAMIENTO OR- TODÓNTICO}

Este índice se desarrolló por Brook y Shaw, en el año 1989 para pobladores del Reino Unido. Lo denominaron Index of orthodontic Treatment Need: IOTN. Reúne dos componentes: el estético y el de salud. ${ }^{18,19}$

\section{1 Índice de estética dental (DAI)}

Este indicador, es muy utilizado por la OMS, fue desarrollado en 1986 por Cons y Kohout en 1986, incluye criterios de tipo psicosociales, clínicos, se suele emplear en estudios epidemiológicos. ${ }^{20,21}$ (Ver la tabla 2)

"El cálculo de la puntuación global se realiza mediante la fórmula: Ausencia (x 6) + Apiñamiento + Espaciamiento + Diastema (x 3) + Irregularidad superior + Irregularidad inferior + Resalte maxilar (x 2) + Resalte mandibular (x 4) + Mordida abierta anterior (x 4) + Relación molar anteroposterior (x 3) + 13 = Índice Estético Dental."

Existen 4 categorías o intervalos DAI su forma de aplicar y su análisis está ampliamente descrita en el manual para realizar encuestas epidemiológicas de salud bucal de la OMS (20).Una limitación del DAI, es que no toma en cuenta algunas características oclusales como son las mordidas anterior y posterior, abierta y profunda y mordidas cruzadas, además de las desviaciones de líneas medias. ${ }^{22,23}$

\section{CALIDAD DE VIDA RELACIONADA A LA SALUD BUCAL}

La salud bucodental relacionada con la calidad de vida del paciente sea descrito como un problema en la salud bucodental o las enfermedades bucodentales, el bienestar o la calidad de vida en general. Las enfermedades orales pueden tener una afectación negativa en la vida de los infantes en edad preescolar, afectando su crecimiento, el peso, la socialización, la autoestima, el aprendizaje, y también en la calidad de vida de sus padres. En estudios de meta-análisis sobre el impacto social del bullying relacionado con las maloclusiones se concluye que las víctimas tienden a aislarse socialmente, a sufrir de problemas psicológicos como la ansiedad y la depresión. ${ }^{24}$ Hoy en día hay poca información sobre el impacto negativo que causan las enfermedades bucales en la vida cotidiana y la calidad de vida del individuo; es por eso que diferentes autores han desarrollado investigaciones en distintas poblaciones que comprueban la íntima relación de la salud bucal y la calidad de vida de los pacientes. ${ }^{25}$

\section{IMPLICANCIA DE LAS MALOCLUSIONES EN CALIDAD DE VIDA}

A continuación, aspectos en los que influyen las maloclusiones en la calidad de vida: 
Tabla 1. Intrumentos validados para medir la Calidad de Vida Relacionada a la Salud Bucal en niños

\begin{tabular}{|c|c|c|c|}
\hline Referencia & Nombre del instrumento & Entrevistado & Edad (albo) años \\
\hline $\begin{array}{l}\text { Locker et al., } \\
2002 \text { (14) }\end{array}$ & $\begin{array}{l}\text { Family Impact Scale (FIS) } \\
\text { Parental / Caregivers }\end{array}$ & Padres & $\begin{array}{l}\text { Padres de niños entre } \\
2 \text { a } 14 \text { años de edad }\end{array}$ \\
\hline $\begin{array}{l}\text { Jokovic et al., } \\
2003 \text { (15) }\end{array}$ & $\begin{array}{l}\text { Perceptions Questionnaire } \\
\text { (P-CPQ) }\end{array}$ & Padres & $\begin{array}{l}\text { Padres de niños entre } \\
6 \text { a } 14 \text { años de edad }\end{array}$ \\
\hline $\begin{array}{l}\text { Pahel et al., } \\
2007 \text { (1) }\end{array}$ & $\begin{array}{l}\text { Early Childhood Oral } \\
\text { Health Scale (ECOHIS) }\end{array}$ & Padres & $\begin{array}{l}\text { Padres de niños entre } \\
2 \text { a } 5 \text { años de edad }\end{array}$ \\
\hline $\begin{array}{l}\text { Jokovic et al., } \\
2002 \text { (2) }\end{array}$ & $\begin{array}{l}\text { Child Perceptions } \\
\text { Questionnaire (CPQ 6-7) }\end{array}$ & Niños & $\begin{array}{l}\text { Niños entre } 6 \text { a } 7 \\
\text { años de edad }\end{array}$ \\
\hline $\begin{array}{l}\text { Jokovic et al., } \\
2004 \text { (17) }\end{array}$ & $\begin{array}{l}\text { Child Perceptions } \\
\text { Questionnaire (CPQ 8-10) }\end{array}$ & Niños & $\begin{array}{l}\text { Niños entre } 8 \text { a } 10 \\
\text { años de edad }\end{array}$ \\
\hline $\begin{array}{l}\text { Jokovic et al., } \\
2006 \text { (18) }\end{array}$ & $\begin{array}{l}\text { Short Child Perceptions } \\
\text { Questionnaire (CPQ 11-14) }\end{array}$ & Niños & $\begin{array}{l}\text { Niños entre } 11 \text { a } 14 \\
\text { años de edad }\end{array}$ \\
\hline $\begin{array}{l}\text { Gherunpong et } \\
\text { al., } 2004 \text { (19) }\end{array}$ & $\begin{array}{l}\text { Child Oral Impact on Daily } \\
\text { Performances Index (Child- } \\
\text { OIDP) }\end{array}$ & Niños & $\begin{array}{l}\text { Niños entre } 11 \text { a } 14 \\
\text { años de edad }\end{array}$ \\
\hline
\end{tabular}

\section{Alteraciones dentofaciales Ponderación}

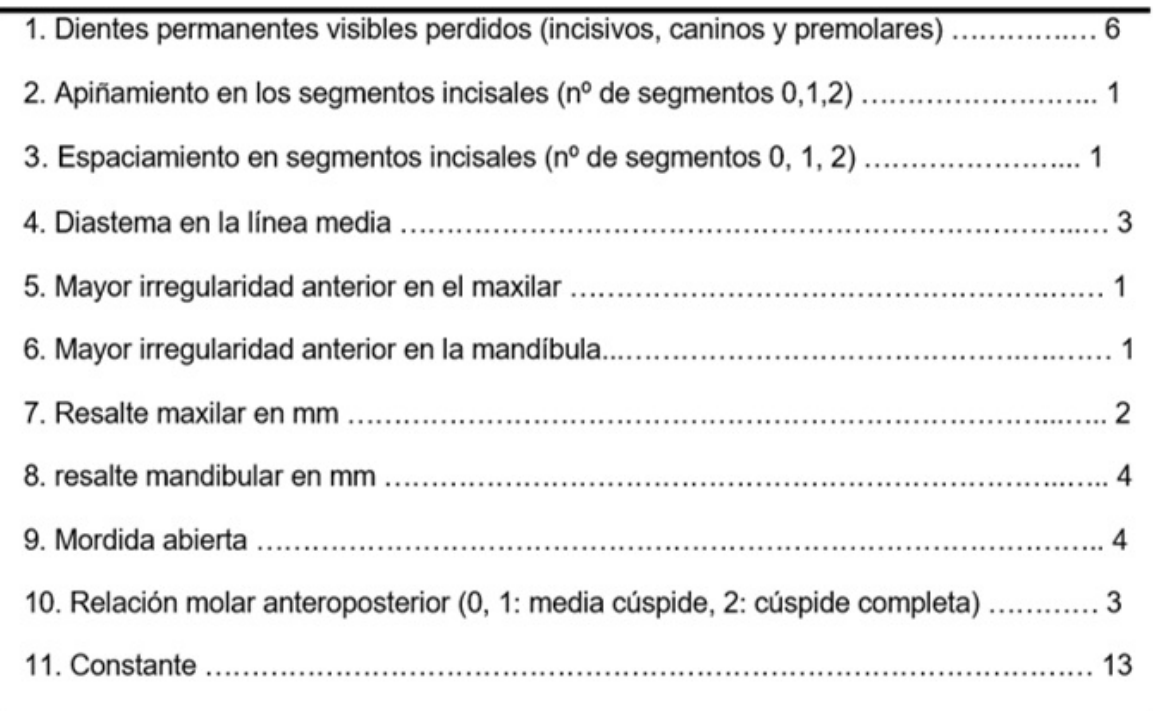

Tabla 2. Parámetros y ponderación utilizados en el índice Estético Dental

\subsection{Aspecto funcional}

Aunque el impacto de las maloclusiones sobre la calidad de vida no sea totalmente comprendido, se sabe que ciertos tipos de maloclusiones como mordidas abiertas, maloclusiones de Clase II de Angle con gran protrusión de los dientes incisivos o mordida profunda y maloclusiones de Clase III de Angle con mordida cruzada posterior o lateral puede contribuir al desarrollo de patología de la articulación temporomandibular a largo plazo. $^{26}$

\subsection{Aspecto psicosocial}

La autopercepción de la maloclusión es muy difícil de medir y definir, debido a que está condicionada por condiciones culturales, étnicas y tendencias de la estética. ${ }^{27}$ Distintos estudios transversales defienden que las personas satisfechas con su apariencia facial son más seguras de sí mismas y tienen una autoestima más alta que los que están insatisfechos con su apariencia facial. Como parte de la apariencia facial, los dientes tienen un papel de gran importancia porque hay una preocupación con su alineamiento y su apariencia y, la 
Tabla 3. Interrelación entre necesidad de salud bucal y calidad de vida relacionada con la misma

INTERRELACIÓN ENTRE NECESIDAD DE SALUD BUCAL Y CALIDAD DE VIDA RELACIONADA CON LA MISMA.

Figura 1: Interrelación entre necesidad de salud bucal y calidad de vida relacionada con la misma.

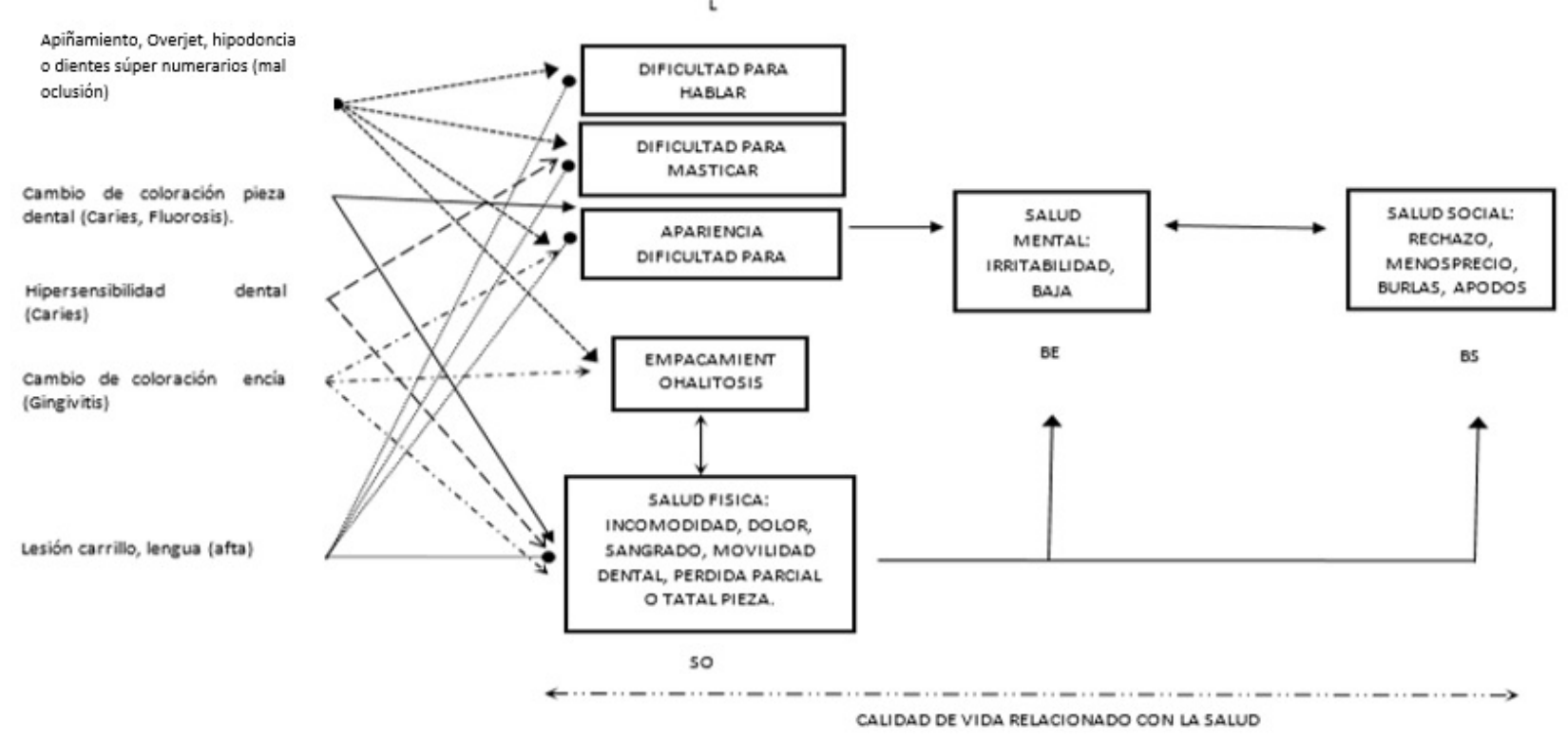

LF= LIMITACIÓN FUNCIONAL, SO= Sintomatología Oral, BE= Bienestar Emocional, BS = Bienestar Social.

maloclusión puede tener impacto en la apariencia general del rostro cambiando la estética. ${ }^{28}$ Sin embargo, el problema de la apariencia, no siempre afecta al individuo.

\subsection{Aspecto académico}

Estudios refieren que las maloclusiones generan vergüenza y esta puede influir en el rendimiento académico en la escuela, que posteriormente afecta la toma de decisiones como la elección de la ocupación o profesión. ${ }^{29} \mathrm{La}$ adolescencia se caracteriza por ser un momento de la vida en la que la auto imagen se ve distorsionada y existe una sobre reacción a los problemas de la apariencia personal. La autopercepción está reflejada en como la persona maneja su entorno o calidad de vivir y en su entorno social y los valores que aplica, contrastando con sus objetivos, expectativas de vida y preocupaciones. ${ }^{30}$

\section{DISCUSIÓN}

Es indispensable tener en cuenta que muchos de los análisis usados en la ortodoncia, están basados en cefalometría extranjera, lejos de las medidas para un tratamiento en la población andina, cuya morfología varía de la extrajera. Respecto del Perú, cabe resaltar que a pesar de la predominancia de nativos y mestizos nativos, son muy escasos los estudios antropométricos de la zona andina peruana y menos de la población en Puno, siendo: Taylor y cols en el 2009 expresaron que la calidad de vida no solo puede estar afectada por las mal oclusiones, ya que en su estudio demostraron que la mayoría de sus pacientes tenía una aceptable calidad de vida pero esta podría mejorar con tratamiento de su defecto; pero sin embargo llegaron a la conclusión que las maloclusiones no es un factor determinante que afecte la calidad de vida pero si puede mejorar en gran manera la percepción del individuo sobre esta. Gherunpong en su estudio en niños de Thailandia del 2004 concluyeron que las alteraciones psicosociales se dan por la maloclusion, y que uno de los motivos de mayor impacto es la vergüenza de sonreír por malas posiciones dentales. Este estudio manifiesta que la severidad de las maloclusiones no tiene que ver o no esta asociada con la calidad de vida de la persona pero estas maloclusiones sí podrían afectar en el bienestar emocional. Mientras que Malandris , Mahoney en un estudio en el 2006 
asegura que las enfermedades orales pueden tener un impacto perjudicial en la vida de los infantes en edad preescolar, afectando su crecimiento, el peso, la socialización, la autoestima, el aprendizaje, y también en la calidad de vida de sus padres. ${ }^{31}$ Acerca de la consideración del aspecto estético de cada persona Alijard J. 1983 considera: No a todos los individuos les afectan las maloclusiones en la calidad de vida en un aspecto psicosocial derivado a estética. ${ }^{32}$ Pero tenemos que tener en cuenta que la apariencia física cumple un rol importante en nuestro medio ya que es considerada muy importante para conseguir un buen trabajo, además tener la aceptación de la sociedad. ${ }^{33}$

\section{CONCLUSIONES}

La zona andina, no tiene parámetros de tratamiento estandarizados según la cefalometría de su población, lo que puede llevar al fracaso del tratamiento ortodóntico. Es indispensable la investigación acerca del tema, de manera que podamos reforzar la importancia de incluir en los servicios de salud en nuestra población andina, tratamientos ortodónticos a la medida de nuestra población, puesto que estos influyen en muchos aspectos en la calidad de vida de los individuos que sufren maloclusiones, pudiendo ser partes de su futuro éxito personal y profesional. La importancia de brindar tratamientos ortodónticos se da más aún en niños y adolescentes, en quienes la patología aún está en desarrollo, pudiendo intervenir antes de llegar a tratamientos complejos y costosos. Existe también una gran necesidad de que cada profesional de salud, aplique sus conocimientos en cada paciente, considerándole íntegramente como un ser individual con rasgos y personalidad únicas, apostado por una mejor calidad de vida para cada uno de ellos.

Conflicto de intereses y financiamiento Los autores declaran no tener conflicto de , haber cumplido con los requisitos de autoría y haber autofinanciado este artículo.

\section{Referencias}

1 Vellard J. Manual de antropologia fisica. Perú: Instituto Riva Aguero; 1959.

2 Cañarte M. Análisis Cefalometrico de Steiner y estudio radiogrfico en pacientes de raza mestiza con maloclusion tipo II de Angle. Tesis doctoral. Guayaquil: Universidad de Guayaquil, Facultad Piloto de Odontologia; 2011.

3 Brusola J. Ortodoncia Clinica y Terapeutica. España ed.: Masson; 2000.

4 Orellana O, Mendoza J, Perales S, Marengo H. Estudio descriptivo de todas las investigaciones sobre prevalencia de mal oclusiones realizadas en las universidades de Lima, ica y arequipa. Odontología Sanmaquina. 2000; 1(5): p. 39-43.

5 Onyeaso C. Prevalence of maloclussion among adolescent in Ibandan Nigeria. Am J Orthod Dentofacial Orthop. 2004; 126: p. 605-607.
6 Isper A, Pantaleao S, Goncalves P. Prevalencia de maloclusion en la denticion primaria en el municipio de Cceres Brasil. Revista Cubana estomatologica. 2007; 45(1): p. 9195.

7 Jokovic A, Locker D, Stephens M, Kenny D. Valiof and Reability of a questionnaire for measuring child oral healthrealated quality life. J Dent Res. 2002; 81(7): p. 59-63.

8 Abanto A, Beneker M, Raggio D. Impacto de los problemas bucales sobre la calidad de vida de niños. Revista Estomatologica Herediana. 2010; 20(1): p. 38-43.

9 Talekar B, Rozier R, Zeldin L. Spanish version of the early childhood oral health Impact Scale. J Dent Res. 2005; 84(1).

10 Bernabe E, Sheiham A, Tsakos G. A comprehensive evaluation of the validity og Child-OIDP: further evidence from Peru. Community Dent Oral Epidemiol. 2008; 36(4).

11 Alvarado R. Prevalencia y Necesidad de tratamiento de caries dental en la I.E Reina de España N 7053. BarrancoLima. Tesis de Bachiller de cirujano dentista. Lima: UNMSM, Facultad de Odontologia; 2005.

12 Echarri P. Diagnostico en Ortodoncia estudio multidisciplinario.. Quintessence. 1998; 5(1).

13 LLanta Abreu M, Grau A. Calidad de vida relacionada con la salud en Oncopediatría: hacia una reflexión del tema. Revista Habanera de Ciencias Medicas. 2014; 13(3): p. 528536.

14 Alvarado R. Prevalencia y necesidad de tratamiento de caries dental de la I.E Reina de España. Tesis de Bachiller cirujano dentista. Lima: UNMSM, Facultad de Odonrología; 2005. Report No.: 7053.

15 Jhonson H. Statistics Applied to Dentistry. Documiento Mimeografico. 1950.

16 Brook P, Shaw W. The development of an index of orthodontic tratment priority. European Journal of Orthodontics. 1989; 11(3).

17 Grabert T, Vanarsdall R, Vig K. Ortodoncia: Principios y tecnicas actuales. Cuarta ed.: Elsevier; 2006.

18 Gutierrez C, Martin C. Estudio epidemiologico de maloclusiones en niños de 6 a 15 años de la comunidad de Madrid de acuerdo con el indice estetico dental: Comparacion entre dos grupos. Tesis doctoral. Madrid: Universidad de Complutense de Madrid, Departamento de Odontología ; 2008.

19 A M, Barrera D, Muñoz G. Índice de estetica dental em adolescentes de San Juan de Pasto. Tesis Doctoral. Pasto: Universidad cooperativa de Pasto- Colombia, Departamento de Odontología ; 2009.

20 Ginebra. Encuesta de Salud Bucodental. Métodos básicos. Cuarta ed. OPS-OMS , editor.; 1997.

21 Bellot Arcis C. Necesidad de tratamiemto ortodóncico en la poblacion aduta de la comunidad Valenciana. [Online].; 2011.

22 Bernabe E, Flores M. Orthodontic tratment need in peruvian young adults evaluated through dental Aesthetic Idex. Angle Orthodontist. 2006; 7(3): p. 417-421.

23 Zhang M, Mcgrath C, Hagg U. The impact of maloclussion 
and its treatment on quality of life: a literature review. Int $\mathbf{J}$ Paediatr Dent. 2006; 16(1): p. 381-387.

24 Allen P. Assesment of oral health related quality of life.. Health Qual Life Outcomes. 2003; 1(40).

25 Alijard J. Planificacion de prioridad en los tratamientos de las anomalias dentofaciales. Ort Esp. 1983; 1(27): p. 65-75.

26 Galarraga D. Motivo de consulta en el paciente ortodoncico adolescente. Acta Odontol Venez. 2000; 38(1).

27 Salas M, Aguilar F, Arguello M, Montero A. Prevalencia de maloclusiones en adolescentes de 15 años del gran área metropolitana: Analisis de modelos. 2005; 1

28 Bellagamba H. Infantile psycology applied to orthodontic. Arsh Dis child. 2002 Marzo; 23(17).

29 Bernael S. Retazos en la constitucion de un sueño pedagógico. Rev Fed Odontol Colombia. 2000; 59(198): p. 45-73.

30 Broder H. Childrens oral health-related quality of life. Community Dent Oral Epidemiol. 2007; 35(1): p. 5-7.

31 Cubilla J. Determinacion del corte optimo para tamizaje de necesidades de salud bucsal del CPQ 11-14 en escolares mexicanos. 2014; 2(1).

32 Malandris M, Mahoney. A etiology, diagnosis and treatment of posterior cross-bites in the primary dentition. Int. J pediatric Dent. ; 14(1): p. 155-166.

33 Alves CNE. Actualizacion de Ortodoncia y Ortopedia Funcional de los maxilares. Artes medicas. 2002; 3(1).

Recibido: 1 de Octubre de 2016

Aceptado: 2 de Diciembre de 2016 